\title{
Comparative performance of reference laboratory tests and in-clinic tests for Giardia in canine feces
}

\author{
Jennifer Mizhquiri Barbecho, Dwight D. Bowman* and Janice L. Liotta
}

\begin{abstract}
Background: We examined the performance of four in-clinic Giardia diagnostic tests by comparing results to three laboratory methods for detection of Giardia. A set of 177 fecal samples originally submitted to a commercial laboratory by veterinarians for routine ova and parasite (O\&P) testing was used. Specimens were examined by direct immunofluorescence assay (DFA) for presence of Giardia cysts which served as the gold standard. Fecal samples were tested using a Giardia-specific cyst wall antigen microtiter plate format enzyme-linked immunosorbent assay (ELISA) and each of the in-clinic assays adhering to the package insert for each kit.

Results: Evaluated were four in-clinic antigen test kits: VetScan ${ }^{\oplus}$ Canine Giardia Rapid Test (Abaxis), Anigen ${ }^{\oplus}$ Rapid CPV-CCV-Giardia Antigen Test (BioNote), SNAP® Giardia Test (IDEXX) and Witness ${ }^{\oplus}$ Giardia Test (Zoetis). In the comparison of the in-clinic tests to the DFA standard test sensitivity ranged between $70.0-87.1 \%$, and specificity ranged between $71.1-93.4 \%$.

Conclusion: Of the tests evaluated here, the SNAP test had the highest sensitivity and specificity. The SNAP test had the highest percent positive and percent negative agreement when compared to the microtiter plate format ELISA and the O\&P assay.
\end{abstract}

Keywords: Giardia, Giardia diagnostics, Fecal assay, Antigen assay, In-clinic diagnostics, Point of care diagnostics, Canine disease

\section{Background}

Giardia are protozoan parasites capable of infecting numerous mammals including both dogs and cats and has been associated with gastrointestinal disease [1-3]. For dogs, diarrhea is a common clinical manifestation presented to veterinary clinics and a consideration in managing these patients is determining Giardia infection status. Giardia can be identified in feces by visualization of whole cysts by microscopy using zinc sulfate $\left(\mathrm{ZnSO}_{4}\right)$ fecal floatation [ova and parasite $(\mathrm{O} \& \mathrm{P})$ test], by direct immunofluorescence (DFA) using Giardia-specific fluorescent antibodies or by demonstrating the presence of Giardia-specific antigen in feces using an immunoassay [4-6].

Fecal flotation can be performed in-clinic but identification of cysts can be difficult because of yeast and

\footnotetext{
* Correspondence: DDB3@cornell.edu

College of Veterinary Medicine, Cornell University, C4-114 VMC, 930 Campus Rd, Ithaca, NY 14853, USA
}

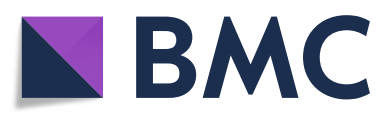

(c) The Author(s). 2018 Open Access This article is distributed under the terms of the Creative Commons Attribution 4.0 International License (http://creativecommons.org/licenses/by/4.0/), which permits unrestricted use, distribution, and

reproduction in any medium, provided you give appropriate credit to the original author(s) and the source, provide a link to the Creative Commons license, and indicate if changes were made. The Creative Commons Public Domain Dedication waiver (http://creativecommons.org/publicdomain/zero/1.0/) applies to the data made available in this article, unless otherwise stated.

debris in the fecal sample which resemble cysts and because shedding is intermittent requiring multiday examinations [7]. Direct immunofluorescence assay has been shown to be more sensitive and specific than conventional flotation methods and herein served as the gold standard method for identifying fecal Giardia $[5,8,9]$. The DFA requires a fluorescent microscope limiting its usefulness in-clinic.

Giardia antigen detection tests detect a secreted soluble Giardia-specific cyst wall antigen and are available from several sources $[3,6,7]$. These tests are available as microtiter plate format ELISAs which can be read visually or by an instrument-based procedure in a reference laboratory setting and single use rapid tests which are read visually and are used in-clinic. A number of in-clinic tests have been described and are now available for use but studies directly comparing the performance of these commercially available tests have not been reported $[6,10,11]$. Using DFA as the gold standard, we examined the 
performance of four in-clinic rapid tests using a set of clinic samples originally submitted to a commercial laboratory by veterinarians for conventional ova and parasite (O\&P) testing. Evaluated were four in-clinic antigen test kits: VetScan ${ }^{\oplus}$ Canine Giardia Rapid Test (Abaxis), Anigen ${ }^{\circ}$ Rapid CPV-CCV-Giardia Antigen Test (BioNote), SNAP ${ }^{\circ}$ Giardia Test (IDEXX) and Witness ${ }^{\circ}$ Giardia Test (Zoetis).

\section{Methods}

\section{Canine samples}

Fecal samples were sourced from IDEXX Reference Laboratories (IRL) by the sole criterion that they were submitted by practicing veterinarians for general ova and parasite $(\mathrm{O} \& \mathrm{P})$ testing which was performed at the reference laboratory using $\mathrm{ZnSO}_{4}$ centrifugal floatation. No clinical information was available. Samples were stored frozen $\left(-20{ }^{\circ} \mathrm{C}\right)$ after the initial O\&P test. A total of 87 O\&P positive and 90 O\&P negative samples was randomly selected so approximately an equal number of O\&P positive and negative samples were obtained. Technicians performing all remaining testing had no knowledge of the O\&P results.

\section{In-clinic tests}

Evaluated were four in-clinic antigen rapid test kits: VetScan ${ }^{\circ}$ Canine Giardia Rapid Test (Abaxis, Union City, USA), Anigen ${ }^{\circ}$ Rapid CPV-CCV-Giardia Antigen Test (BioNote, Seoul, Korea), SNAP ${ }^{\circ}$ Giardia Test (IDEXX, Westbrook, USA) and Witness Giardia Test (Zoetis, Parsippany, USA). The four kits were tested concurrently adhering to the package insert for each kit. Before testing samples were randomized and blind-labeled. There were no additional freeze-thaw cycles or sample handling differences in testing events between the four rapid tests. Visual interpretation of results was performed following the manufacturers' instructions supplied with each test kit.

\section{Microtiter plate format ELISA}

Samples were tested with the ProSpecT ${ }^{\oplus}$ Giardia/Cryptosporidium Microplate Assay (Thermo Fisher Scientific, Pittsburgh, USA) using instructions supplied with the kit. Plates were read spectrophotometrically at $450 \mathrm{~nm}$; samples were considered positive when the net optical density reading (sample OD-negative control OD) was $\geq 0.05$.

\section{DFA test}

The DFA was conducted and Giardia cysts were counted using a previously described procedure with modifications [5]. Briefly, $0.1 \mathrm{~g}$ feces was added to $900 \mu \mathrm{l}$ $0.02 \mathrm{M}$ sodium phosphate-buffered saline $\mathrm{pH}$ 7.4 (PBS) and mixed thoroughly. An aliquot of the mixed sample $(100 \mu \mathrm{l})$ was added to a second tube containing $900 \mu \mathrm{l}$ of PBS. The second tube was thoroughly mixed and 100 $\mu \mathrm{l}$ was added to a third tube which was mixed with $5 \mu \mathrm{l}$ of Merifluor $^{\circ}$ detection reagent (Meridian Biosciences, Cincinnati, USA). The sample was then incubated at room temperature for $30 \mathrm{~min}$ in the dark and then maintained at $4{ }^{\circ} \mathrm{C}$ until the quantitative cyst count was performed. A sample volume of $10.5 \mu \mathrm{l}$ was removed and examined using a fluorescent microscope. The cysts were counted and a total cysts/gram was calculated. A positive and negative control were read prior to each test. The positive control was $85 \mu \mathrm{l}$ PBS, $15 \mu \mathrm{l}$ positive control from the Merifluor kit, and $5 \mu \mathrm{l}$ Merifluor detection reagent. The negative control consisted of $100 \mu \mathrm{l}$ PBS and $5 \mu \mathrm{l}$ Merifluor detection reagent.

\section{Data collection and analysis}

Statistical analysis was performed in SAS৫ version 9.4 (SAS Institute, Inc., Cary, NC, USA). Agresti-Coull 95\% confidence intervals were used for calculation of sensitivity and specificity compared to the DFA reference method, as well as percent positive and negative agreement to the microtiter plate format ELISA test. Agresti-Coull confidence intervals were calculated using the FREQ procedure in SAS 9.4. Prevalence adjusted test agreement was calculated using the following formula: agreement $=$ preva lence $\times$ sensitivity $+(1-$ prevalence $) \times$ specificity. In this calculation, we used the prevalence (5.4\%) found for client-owned symptomatic dogs presented to a veterinary hospital as determined by a monoclonal antibody-based immunofluorescent antibody assay [12].

\section{Results \\ DFA, O\&P and microtiter plate ELISA results}

The final sample set consisted of 177 samples; all were examined by DFA and by microtiter plate format ELISA. Cysts were identified by DFA in 101 of 177 samples which were classified as positive and were not identified in 76 samples which were classified as negative. DFA results were compared to the original O\&P results reported by the reference lab. In the O\&P test, cysts

Table 1 Statistical comparison of ova and parasite results and reference laboratory ELISA to DFA with calculated sensitivity and specificity

\begin{tabular}{llll}
\hline Reference laboratory test & Sensitivity $(95 \% \mathrm{Cl})$ & Specificity $(95 \% \mathrm{Cl})$ & Prevalence adjusted $\%$ agreement $^{\text {a }}$ \\
\hline Ova and Parasite (O\&P) Test & $81.2(72.4-87.7)$ & $93.4(85.2-97.5)$ & 92.7 \\
ProSpecT Microtiter Plate ELISA & $94.1(87.4-97.5)$ & $97.4(90.4-99.8)$ & 97.2
\end{tabular}

${ }^{\mathrm{a}}$ Percent agreement was adjusted for reported prevalence of infection determined by DFA as described in materials and methods 
Table 2 Statistical results of four in-clinic antigen tests for Giardia using DFA as the reference standard with calculated sensitivity and specificity

\begin{tabular}{llll}
\hline In-clinic test & Sensitivity $(95 \%$ Cl) & Specificity (95\% Cl) & Prevalence adjusted \% agreement $^{a}$ \\
\hline SNAP Giardia & $87.1(79.1-92.5)$ & $93.4(85.2-97.5)$ & 93.1 \\
Anigen Rapid CPV-CCV-Giardia Antigen Test & $80.2(71.3-86.9)$ & $80.3(69.8-87.8)$ & 80.3 \\
Witness Giardia Test & $73.3(63.9-81.0)$ & $71.1(60.0-80.1)$ & 71.2 \\
VetScan Canine Giardia Rapid Test & $70.00(60.4-78.1)$ & $85.5(75.7-91.9)$ & 84.7 \\
Ova and Parasite Test & $81.2(72.4-87.7)$ & $93.4(85.2-97.5)$ & 92.7 \\
\hline
\end{tabular}

${ }^{a}$ Percent agreement was adjusted for reported prevalence of infection determined by DFA as described in materials and methods

were identified in 82 of the 101 DFA positive samples (sensitivity $81.2 \%$ ) and in 5 of the 76 DFA negative samples (specificity 93.4\%). In the microtiter plate format ELISA, 95 of the 101 DFA reactive samples were positive (sensitivity 94.1\%) and 74 of the 76 DFA negative samples were negative (specificity 97.4\%). A comparison of results for the O\&P and microtiter plate test are shown in Table 1.

\section{In-clinic test results}

Among the in-clinic test results, there was a single invalid result with Abaxis VetScan (positive control line did not appear) and therefore there were only $176 \mathrm{com}$ parisons with this test. In all other cases, there were 177 valid results. The in-clinic tests results were compared to results of DFA (Table 2). Sensitivity ranged from $70.0 \%$ for the VetScan test to $87.1 \%$ for the SNAP test, specificity ranged from $71.0 \%$ for the Witness test to 93.4\% for the SNAP test and prevalence adjusted agreement ranged from $71.2 \%$ for the Witness test to $93.0 \%$ for the SNAP test. In-clinic test results were also compared to results of the microtiter plate format ELISA, values for positive and negative agreement and overall agreement are shown in Table 3. Among the in-clinic rapid tests, SNAP Giardia showed the highest agreement with DFA and the microtiter plate format ELISA.

\section{Discussion}

We sought to compare the performance of four commercially available in-clinic assays designed to detect Giardia infection in dogs. Although we used the O\&P test to select samples to ensure a sufficient population of negative and positives, we used direct immunofluorescence as the gold standard because several studies have demonstrated its accurate performance compared to traditional flotation methods. We chose to include the microtiter plate format test for comparison because the test detects soluble Giardia specific cell wall antigen shed in the local environment and is used for high throughput screening in reference laboratories. In comparing the microtiter plate format ELISA results to the DFA results, ELISA had a sensitivity of $94.1 \%$, and a specificity of $97.4 \%$ (Table 1 ).

Results for the comparison of the in-clinic tests to the DFA and the microtiter plate format ELISA standard are shown in Tables 2 and 3, respectively. In all three measures of performance, SNAP had the highest concordance with the DFA and the ELISA standards among the tests.

We also used DFA results to retrospectively evaluate performance characteristics of the O\&P method (Table 1) and compared these to those found for the 4 in-clinic tests compared to DFA shown in Table 2. We observed that the performance of SNAP Giardia was in close agreement with the O\&P method while each of the 3 remaining in-clinic tests showed reduced values for percent positive agreement, percent negative agreement and prevalence adjusted agreement relative to this method.

The performance of SNAP Giardia has been studied by several groups $[6,10,11]$. To our knowledge, this is the first study to evaluate multiple tests currently available for detection of Giardia including several new rapid in-clinic tests. We found the performance of rapid in-clinic tests varied by comparing to different reference standards. There could be a myriad of factors attributable to the observed variability. The SNAP Giardia is an ELISA-based assay designed for in-clinic use and the methodology has been described in detail in a recent technical review [13]. In the

Table 3 Statistical results of four in-clinic antigen tests for Giardia using ProSpecT ELISA Giardia Test as the reference standard with calculated positive and negative percent agreement

\begin{tabular}{llll}
\hline In-clinic test & \% Positive agreement (95\% Cl) & \% Negative agreement (95\% Cl) & Prevalence adjusted \% agreement $^{\text {a }}$ \\
\hline SNAP Giardia & $91.8(84.4-96.0)$ & $95.0(87.5-98.4)$ & 94.8 \\
Anigen Rapid CPV-CCV-Giardia Antigen Test & $80.4(71.3-87.2)$ & $77.5(67.1-85.4)$ & 77.7 \\
Witness Giardia Test & $77.3(68.0-84.6)$ & $73.8(63.1-82.2)$ & 74.0 \\
VetScan Canine Giardia Rapid Test & $72.9(63.2-80.8)$ & $86.3(76.9-92.3)$ & 85.6 \\
\hline
\end{tabular}

apercent agreement was adjusted for reported prevalence of infection determined by DFA as described in materials and methods 
same journal issue dealing with "Point of Care Tests in Veterinary Medicine", the lateral flow methodology, which is the basis for the other three in-clinic tests, is also described in detail [14]. Many specific components of each individual test are proprietary. It is not clear what specific differences in the in-clinic assayed may have caused the observed performance differences between the tests.

\section{Conclusions}

We found that results of rapid in-clinic Giardia assays can vary amongst tests. Overall, of the in-clinic tests evaluated, the SNAP test most closely mirrored the results of the DFA and the microtiter plate ELISA and was the only test with a prevalence adjusted agreement greater than the O\&P method. The major conclusion of this study is that among the in-clinic tests evaluated, Giardia SNAP is the most reliable method for detection of Giardia in canine stool samples.

\section{Abbreviations}

DFA: Direct immunofluorescence assay; ELISA: Enzyme-linked immunosorbent assay; IRL: IDEXX Reference Laboratories; O\&P: Ova and parasite testing, zinc sulfate fecal flotation; PBS: Phosphate-buffered saline $0.02 \mathrm{M} \mathrm{pH} 7.4 ; \mathrm{ZnSO}_{4}$ : Zinc sulfate flotation solution

\section{Acknowledgements}

The authors thank IDEXX (Westbrook, ME) for generously providing the canine fecal samples used in this study. IDEXX had no input on the manuscript, results or interpretation of the results.

\section{Funding}

Not applicable.

\section{Availability of data and materials}

Data supporting the conclusions of this article are included within the article. The datasets used or analyzed during the present study are available from the corresponding author upon reasonable request.

\section{Authors' contributions}

$J L L$ and JMB performed experiments and DDB performed analyses. All authors read and approved the final manuscript.

\section{Ethics approval and consent to participate}

Not applicable.

\section{Consent for publication}

Not applicable.

\section{Competing interests}

The authors declare they have no competing interests.

\section{Publisher's Note}

Springer Nature remains neutral with regard to jurisdictional claims in published maps and institutional affiliations.

Received: 25 February 2018 Accepted: 3 July 2018

Published online: 01 August 2018

\section{References}

1. Ballweber LR, Xiao L, Bowman DD, Kahn G, Cama VA. Giardiasis in dogs and cats: update on epidemiology and public health significance. Trends Parasitol. 2010;26:180-9.

2. Bowman DD, Lucio-Forster A. Cryptosporidiosis and giardiasis in dogs and cats: veterinary and public health importance. Exp Parasitol. 2010;124:121-7.
3. Tangtrongsup S, Scorza V. Update on the diagnosis and management of Giardia spp. infections in dogs and cats. Top Companion Anim Med. 2010;25:155-62.

4. Geurden T, Berkvens D, Casaert S, Vercruysse J, Claerebout E. A Bayesian evaluation of three diagnostic assays for the detection of Giardia duodenalis in symptomatic and asymptomatic dogs. Vet Parasitol. 2008;157:14-20.

5. Rishniw M, Liotta JL, Bellosa M, Bowman DD, Simpson KW. Comparison of 4 Giardia diagnostic tests in diagnosis of naturally acquired canine chronic subclinical Giardiasis. J Vet Intern Med. 2010;24:293-7.

6. Uehlinger FD, Naqvi SA, Greenwood SJ, MCClure JT, Conboy G, O'Handley R, Barkema HW. Comparison of five diagnostic tests for Giardia duodenalis in fecal samples from young dogs. Vet Parasitol. 2017;244:91-6.

7. Decock C, Cadiergues MC, Larcher M, Vermot S, Franc M. Comparison of two techniques for diagnosis of giardiasis in dogs. Parasite. 2003;10:69-72.

8. Mekaru SR, Marks SL, Felley AJ, Chouicha N, Kass PH. Comparison of direct immunofluorescence, immunoassays, and fecal flotation for detection of Cryptosporidium spp. and Giardia spp. in naturally exposed cats in 4 northern California animal shelters. J Vet Intern Med. 2007;21:959-65.

9. Rimhanen-Finne R, Enemark HL, Kolehmainen J, Toropainen P, Hanninen ML. Evaluation of immunofluorescence microscopy and enzyme-linked immunosorbent assay in detection of Cryptosporidium and Giardia infections in asymptomatic dogs. Vet Parasitol. 2007;145:345-8.

10. Carlin EP, Bowman DD, Scarlett JM, Garrett J, Lorentzen L. Prevalence of Giardia in symptomatic dogs and cats throughout the United States as determined by the IDEXX SNAP Giardia test. Vet Ther. 2006;7:199-206.

11. Costa M, Clarke C, Mitchell S, Papasouliotis K. Diagnostic accuracy of two point-of-care kits for the diagnosis of Giardia species infection in dogs. J Small Anim Pract. 2016;57:318-22.

12. Hackett T, Lappin MR. Prevalence of enteric pathogens in dogs of northcentral Colorado. J Am Anim Hosp Assoc. 2003;39:52-6.

13. O'Connor TP. SNAP assay technology. Top Comp An Med. 2015;30:132-8.

14. O'Farrell B. Lateral flow techology for field based applications - basics and advanced developments. Top Comp An Med. 2015;30:139-47.

\section{Ready to submit your research? Choose BMC and benefit from:}

- fast, convenient online submission

- thorough peer review by experienced researchers in your field

- rapid publication on acceptance

- support for research data, including large and complex data types

- gold Open Access which fosters wider collaboration and increased citations

- maximum visibility for your research: over $100 \mathrm{M}$ website views per year

At BMC, research is always in progress.

Learn more biomedcentral.com/submissions 